\title{
Synthesis and Antioxidant Properties of Novel Quinazoline Derivatives
}

\author{
Zenta Tetere $^{1}$, Daina Zicane ${ }^{2}$, Irisa Ravina ${ }^{3}$, Inese Mierina ${ }^{4}$, Inese Rijkure ${ }^{5}$, \\ ${ }^{1-5}$ Riga Technical University
}

\begin{abstract}
Quinazoline derivatives have expressed a broad spectrum of biological activities, but there are few examples in literature on an antioxidant activity of different quinazolines. On the basis of literature by Chinese scientists on the effective antioxidant activity of Schiff bases containing 2-oxoquinoline and due to structural similarity of quinoline and quinazoline ring systems, the reactions of 3-aminoquinazolinone with a range of aromatic aldehydes and ethoxymethylene derivatives have been carried out and their radical scavenging activities have been studied.
\end{abstract}

Keywords - 3-amino-quinazolin-4(3H)-one, aldehydes, antioxidant activity, ethoxymethylene compounds, Schiff bases.

\section{INTRODUCTION}

Quinazoline derivatives have attracted interest over the years because of their various biological activities [1], but there are few examples in literature on antioxidant activity of different quinazolines. Among several quinazolines reported, there are some subtituted quinazolines exhibiting interesting pharmaceutical activities. The antioxidant activity of the molecule may be due to the presence of hydroxyl groups [2] and sulphur atom [3], [4], but no data on systematic studies of quinazoline substituents and antioxidant activity relationships are available.

Recently, Chinese scientists reported [5] an effective antioxidant activity of Schiff bases containing 2-oxoquinoline. Due to the structural similarity of quinoline and quinazolinone ring systems, 4-oxoquinazoline Schiff bases may be viewed as potential antioxidants.

The above-mentioned issue, as well as an increasing necessity and interest in antioxidants as a major defence against damages caused by free radicals, has prompted the synthesis of some derivatives of quinazolin-4(3H)-ones and evaluation of their antioxidative activity.

\section{RESULTS AND DISCUSSION}

The starting compound 3-aminoquinazolin-4(3H)-one (1) was synthesized from anthranilhydrazide and triethyl orthoformate as reported in literature [6].

The starting aminoquinazolinone 1 was modified at $\mathrm{N}(3)$ to yield compounds with $\mathrm{C}=\mathrm{C}$ and $\mathrm{C}=\mathrm{N}$ bonds by reactions of this derivative $\mathbf{1}$ with aromatic and heterocyclic aldehydes $\mathbf{2}$ and ethoxymethylene compounds $\mathbf{3}$ (scheme 1).

The reaction of aminoquinazolinone $\mathbf{1}$ with isopropylidene ethoxymethylenemalonate (3a) proceeds readily even at room temperature. To carry out the reactions with aromatic $\mathbf{2 a - f}$ and heteroaromatic $\mathbf{2 g}$ aldehydes it is necessary to reflux the components $\mathbf{1}$ and $\mathbf{2 a - g}$ for 3 - 6 hours, while to prepare the desired compounds $\mathbf{5 b}$-d the heating of aminoquinazolinone $\mathbf{1}$ with ethoxymethylene derivatives $\mathbf{3 b}$-d in toluene at reflux for long time $(\sim 15 \mathrm{~h})$ is required.<smiles></smiles>

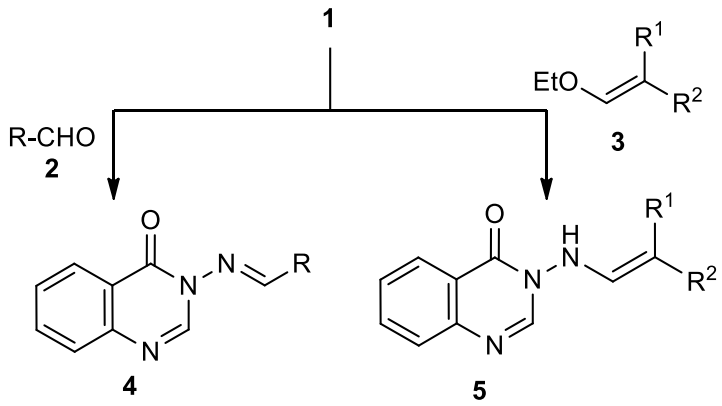

Substituent $\mathrm{R}$ for compounds $\mathbf{2}$ and $\mathbf{4}$<smiles>Clc1ccc([131I])cc1</smiles>
b) -<smiles>Fc1ccc(F)cc1</smiles><smiles>Cc1cccc(F)c1</smilesd)<smiles>Brc1ccccc1Br</smiles>

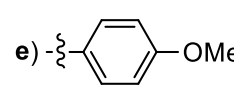<smiles>Oc1ccc(C(F)(F)F)cc1O</smiles><smiles>[AlH2][As]c1cccnc1</smiles><smiles>COc1ccc([Te][Te]c2cc(OC)ccc2OC)c(OC)c1</smiles>
$\mathrm{MeO}$<smiles>COc1cc(OC)c(OC)cc1OC</smiles>

k)<smiles>COc1ccc(I)c(OC)c1OC</smiles><smiles>COc1cc([12F])cc(OC)c1OC</smiles>

Substituents $\mathrm{R}^{1}$ and $\mathrm{R}^{2}$ for compounds $\mathbf{3}$ and $\mathbf{5}$

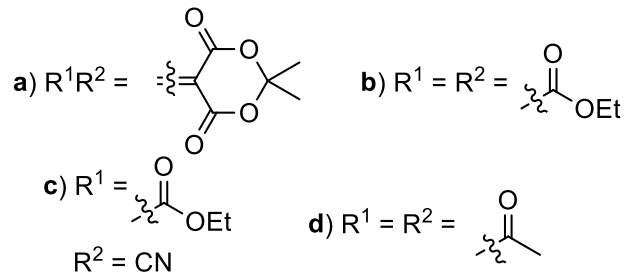

Scheme 1. Synthesis of the derivatives of quinazoline $\mathbf{4}$ and $\mathbf{5}$.

The structure of synthesized compounds was confirmed by the results from elemental analyses (Table 1) and ${ }^{1} \mathrm{H}$ NMR spectra (Table 2) in which signals for the protons of all the structural units were observed in their characteristic ranges. The newly prepared compounds 3a-g, 5a-d as well as previously synthesized compounds 3h-I [7] were evaluated for their antioxidant activities. 
TABLE 1

CHARACTERISTIC OF THE SYNTHESIZED COMPOUNDS 4a-g AND 5a-d

\begin{tabular}{|c|c|c|c|c|c|c|}
\hline \multirow[t]{2}{*}{ Compound } & \multirow[t]{2}{*}{ Empirical formula } & \multicolumn{3}{|c|}{$\frac{\text { Found, } \%}{\text { Calculated, } \%}$} & \multirow[t]{2}{*}{ M. p., ${ }^{\circ} \mathrm{C}$ (recrystallization solvent) } & \multirow[t]{2}{*}{ Yield, \% } \\
\hline & & $\mathrm{C}$ & $\mathrm{H}$ & $\mathrm{N}$ & & \\
\hline $4 a$ & $\mathrm{C}_{15} \mathrm{H}_{10} \mathrm{ClN}_{3} \mathrm{O}$ & $\frac{63.36}{63.50}$ & $\frac{3.51}{3.55}$ & $\frac{14.74}{14.81}$ & $\begin{array}{l}165-168 \\
\text { (ethanol) }\end{array}$ & 75.0 \\
\hline $4 b$ & $\mathrm{C}_{15} \mathrm{H}_{10} \mathrm{FN}_{3} \mathrm{O}$ & $\frac{67.14}{67.41}$ & $\underline{3.67}$ & $\frac{15.68}{15.72}$ & $\begin{array}{l}\text { 160-161 } \\
\text { (ethanol) }\end{array}$ & 98.0 \\
\hline $4 c$ & $\mathrm{C}_{15} \mathrm{H}_{10} \mathrm{FN}_{3} \mathrm{O}$ & $\frac{67.26}{67.41}$ & $\underline{3.61}$ & $\frac{15.66}{15.72}$ & $\begin{array}{c}147-148 \\
\text { (methanol) }\end{array}$ & 51.5 \\
\hline $4 d$ & $\mathrm{C}_{15} \mathrm{H}_{10} \mathrm{BrN}_{3} \mathrm{O}$ & $\frac{54.76}{54.90}$ & $\underline{3.14}$ & $\frac{12.67}{12.80}$ & $\begin{array}{l}165-167 \\
\text { (ethanol) }\end{array}$ & 79.0 \\
\hline $4 e$ & $\mathrm{C}_{16} \mathrm{H}_{13} \mathrm{~N}_{3} \mathrm{O}_{2}$ & $\frac{68.47}{68.81}$ & $\frac{4.70}{4.69}$ & $\frac{15.03}{15.05}$ & $\begin{array}{l}134-135 \\
\text { (ethanol) }\end{array}$ & 82.0 \\
\hline $4 f$ & $\mathrm{C}_{15} \mathrm{H}_{11} \mathrm{~N}_{3} \mathrm{O}_{3}$ & $\frac{64.10}{64.05}$ & $\frac{3.90}{3.94}$ & $\frac{15.01}{14.94}$ & $\begin{array}{c}140-142 \\
\text { (ethyl acetate) }\end{array}$ & 71.0 \\
\hline $4 \mathrm{~g}$ & $\mathrm{C}_{14} \mathrm{H}_{10} \mathrm{~N}_{4} \mathrm{O}$ & $\frac{67.21}{67.19}$ & $\frac{4.07}{4.03}$ & $\underline{22.43}$ & $>250$ & 71.0 \\
\hline $5 \mathbf{a}$ & $\mathrm{C}_{15} \mathrm{H}_{13} \mathrm{~N}_{3} \mathrm{O}_{5}$ & $\underline{57.11}$ & $\underline{3.93}$ & $\frac{13.40}{13.37}$ & $\begin{array}{l}208-210 \\
\text { (ethanol) }\end{array}$ & 78.0 \\
\hline $5 \mathbf{b}$ & $\mathrm{C}_{16} \mathrm{H}_{17} \mathrm{~N}_{3} \mathrm{O}_{5}$ & $\frac{58.03}{58.00}$ & $\frac{5.01}{5.17}$ & $\frac{12.72}{12.68}$ & $\begin{array}{l}131-132 \\
\text { ethanol/water, } 1: 1\end{array}$ & 72.0 \\
\hline $5 c$ & $\mathrm{C}_{14} \mathrm{H}_{12} \mathrm{~N}_{4} \mathrm{O}_{3}$ & $\underline{59.04}$ & $\frac{4.22}{4.25}$ & $\frac{19.97}{19.71}$ & $\begin{array}{l}187-190 \\
\text { (ethanol) }\end{array}$ & 72.0 \\
\hline $5 d$ & $\mathrm{C}_{14} \mathrm{H}_{13} \mathrm{~N}_{3} \mathrm{O}_{3}$ & $\frac{62.03}{61.99}$ & $\frac{4.90}{4.83}$ & $\frac{15.61}{15.49}$ & $\begin{array}{l}\text { 238-239 } \\
\text { (ethanol) }\end{array}$ & 74.0 \\
\hline
\end{tabular}

TABLE 2

${ }^{1} \mathrm{H}$ NMR SPECTRA OF THE SYNTHESIZED COMPOUNDS 4a-g AND 5a-d

\begin{tabular}{|c|c|}
\hline Compound & ${ }^{1} \mathrm{H}$ NMR SPECTRA \\
\hline $4 a$ & $\begin{array}{l}7.49-7.93(4 \mathrm{H}, \mathrm{m}, \operatorname{arom} .) ; 8.18(1 \mathrm{H}, \mathrm{t}, J=5.8, \operatorname{arom} .) ; 8.25(1 \mathrm{H}, \mathrm{d}, J=7.6, \text { arom. }) ; 8.36-8.41(2 \mathrm{H}, \mathrm{m}, \operatorname{arom} .) ; 8.60(1 \mathrm{H}, \mathrm{s},=\mathrm{CH}) ; 9.89(1 \mathrm{H}, \mathrm{s} \text {, } \\
=\mathrm{CH})\end{array}$ \\
\hline $4 b$ & $\begin{array}{l}7.41(2 \mathrm{H}, \mathrm{t}, J=8.8, \text { arom. }) ; 7.61(1 \mathrm{H}, \mathrm{t}, J=7.0, \operatorname{arom} .) ; 7.76(1 \mathrm{H}, \mathrm{d}, J=8.1, \text { arom. }) ; 7.87(1 \mathrm{H}, \mathrm{t}, J=8.8, \text { arom. }) ; 8.02(2 \mathrm{H}, \mathrm{t}, J=8.8, \text { arom. }) ; 8.57 \\
(1 \mathrm{H}, \mathrm{s},=\mathrm{CH}) ; 8.82(1 \mathrm{H}, \mathrm{d}, J=7.0, \text { arom. }) ; 9.31(1 \mathrm{H}, \mathrm{s},=\mathrm{CH})\end{array}$ \\
\hline $4 c$ & $\begin{array}{l}7.47(1 \mathrm{H}, \mathrm{t}, J=7.0, \text { arom. }) ; 7.58-7.79(3 \mathrm{H}, \mathrm{m}, \text { arom. }) ; 7.86(1 \mathrm{H}, \mathrm{t}, J=7.0, \text { arom. }) ; 7.99(2 \mathrm{H}, \mathrm{t}, J=7.0, \text { arom. }) ; 8.24(1 \mathrm{H}, \mathrm{d}, J=7.9, \text { arom. }) ; 8.65 \\
(1 \mathrm{H}, \mathrm{s},=\mathrm{CH}) ; 9.42(1 \mathrm{H}, \mathrm{s},=\mathrm{CH})\end{array}$ \\
\hline $4 d$ & $\begin{array}{l}7.51-7.65(3 \mathrm{H}, \mathrm{m}, \operatorname{arom} .) ; 7.76(1 \mathrm{H}, \mathrm{d}, J=8.1, \text { arom. }) ; 7.80-7.84(1 \mathrm{H}, \mathrm{m}, \text { arom. }) ; 7.90(1 \mathrm{H}, \mathrm{t}, J=8.1, \text { arom. }) ; 8.16-8.20(1 \mathrm{H}, \mathrm{m}, \operatorname{arom} .) ; 8.26(1 \mathrm{H}, \\
\mathrm{d}, J=8.1 \text {, arom. }) ; 8.60(1 \mathrm{H}, \mathrm{s},=\mathrm{CH}) ; 9.88(1 \mathrm{H}, \mathrm{s},=\mathrm{CH})\end{array}$ \\
\hline $4 e$ & $\begin{array}{l}3.86\left(3 \mathrm{H}, \mathrm{s}, \mathrm{CH}_{3}\right) ; 7.12(2 \mathrm{H}, \mathrm{d}, J=8.7, \text { arom. }) ; 7.61(1 \mathrm{H}, \mathrm{t}, J=7.5 \text {, arom. }) ; 7.75(1 \mathrm{H}, \mathrm{d}, J=7.5 \text {, arom. }) ; 7.83-7.94(3 \mathrm{H}, \mathrm{m}, \operatorname{arom} .) ; 8.22(1 \mathrm{H}, \mathrm{d} \text {, } \\
J=7.5 \text {, arom. }) ; 8.54(1 \mathrm{H}, \mathrm{s},=\mathrm{CH}) ; 9.13(1 \mathrm{H}, \mathrm{s},=\mathrm{CH})\end{array}$ \\
\hline $4 f$ & $\begin{array}{l}5.88(2 \mathrm{H}, \mathrm{s}, 2 \mathrm{OH}) ; 7.56(1 \mathrm{H}, \mathrm{t}, J=7.4, \operatorname{arom} .) ; 7.67-7.74(1 \mathrm{H}, \mathrm{m}, \operatorname{arom} .) ; 7.80-7.88(2 \mathrm{H}, \mathrm{m}, \text { arom. }) ; 8.05(1 \mathrm{H}, \mathrm{t}, J=7.7, \operatorname{arom} .) ; 8.18(1 \mathrm{H}, \mathrm{d}, \\
J=7.7 \text {, arom. }) ; 8.26(1 \mathrm{H}, \mathrm{d}, J=8.1 \text {, arom. }) ; 8.36(1 \mathrm{H}, \mathrm{s},=\mathrm{CH}) ; 8.66(1 \mathrm{H}, \mathrm{s},=\mathrm{CH})\end{array}$ \\
\hline $4 \mathrm{~g}$ & $\begin{array}{l}7.56(1 \mathrm{H}, \mathrm{t}, J=7.9, \text { arom. }) ; 7.67-7.74(2 \mathrm{H}, \mathrm{m}, \operatorname{arom} .) ; 7.79(2 \mathrm{H}, \mathrm{m}, \text { arom. }) ; 8.00(1 \mathrm{H}, \mathrm{t}, J=7.9, \text { arom. }) ; 8.18(1 \mathrm{H}, \mathrm{d}, J=7.9, \text { arom. }) ; 8.26(1 \mathrm{H}, \mathrm{d} \text {, } \\
J=8.1 \text {, arom. }) ; 8.34(1 \mathrm{H}, \mathrm{s},=\mathrm{CH}) ; 8.66(1 \mathrm{H}, \mathrm{s},=\mathrm{CH})\end{array}$ \\
\hline $5 a$ & $\begin{array}{l}1.69\left(6 \mathrm{H}, \mathrm{s}, 2 \mathrm{CH}_{3}\right) ; 7.63(1 \mathrm{H}, \mathrm{t}, J=8.0 \text {, arom. }) ; 7.74(1 \mathrm{H}, \mathrm{d}, J=8.1 \text {, arom. }) ; 7.91(1 \mathrm{H}, \mathrm{t}, J=7.1 \text {, arom. }) ; 8.20(1 \mathrm{H}, \mathrm{d}, J=7.1, \text { arom. }) ; 8.47(1 \mathrm{H}, \mathrm{br} . \\
\mathrm{S},=\mathrm{CH}) ; 8.62(1 \mathrm{H}, \text { br. } \mathrm{S},=\mathrm{CH}) ; 11.76(1 \mathrm{H}, \mathrm{s}, \mathrm{NH})\end{array}$ \\
\hline $5 \mathbf{b}$ & $\begin{array}{l}1.26\left(3 \mathrm{H}, \mathrm{t}, J=6.5, \mathrm{CH}_{3}\right) ; 1.37\left(3 \mathrm{H}, \mathrm{t}, J=6.5, \mathrm{CH}_{3}\right) ; 4.21\left(2 \mathrm{H}, \mathrm{q}, J=6.9, \mathrm{CH}_{2}\right) ; 4.33\left(2 \mathrm{H}, \mathrm{q}, J=6.9, \mathrm{CH}_{2}\right) ; 7.56(1 \mathrm{H}, \mathrm{t}, J=7.3, \operatorname{arom} .) ; 7.72-7.86 \\
(2 \mathrm{H}, \mathrm{m} \text {, arom. }) ; 7.94(1 \mathrm{H}, \mathrm{d}, J=11.3,=\mathrm{CH}) ; 8.16(1 \mathrm{H}, \mathrm{s}, \mathrm{NH}) ; 8.30(1 \mathrm{H}, \mathrm{d}, J=7.7 \text {, arom. }) ; 10.61(1 \mathrm{H}, \mathrm{d}, J=11.3,=\mathrm{CH})\end{array}$ \\
\hline $5 c$ & $\begin{array}{l}1.21\left(3 \mathrm{H}, \mathrm{t}, J=6.8, \mathrm{CH}_{3}\right) ; 4.17\left(2 \mathrm{H}, \mathrm{q}, J=7.2, \mathrm{CH}_{2}\right) ; 7.52-7.94(3 \mathrm{H}, \mathrm{m}, \text { arom. }) ; 8.19(1 \mathrm{H}, \mathrm{t}, J=6.4, \text { arom. }) ; 8.37(1 \mathrm{H}, \mathrm{br} . \mathrm{S}, J=7.7,=\mathrm{CH}) ; 8.47 \\
(1 \mathrm{H}, \text { br. S, NH. }) ; 11.18(1 \mathrm{H}, \text { br. } \mathrm{S},=\mathrm{CH})\end{array}$ \\
\hline 5d & $\begin{array}{l}2.19\left(3 \mathrm{H}, \mathrm{s}, \mathrm{CH}_{3}\right) ; 2.40\left(3 \mathrm{H}, \mathrm{s}, \mathrm{CH}_{3}\right) ; 7.62(1 \mathrm{H}, \mathrm{t}, J=8.1, \operatorname{arom} .) ; 7.75(1 \mathrm{H}, \mathrm{d}, J=7.7, \text { arom. }) ; 7.90(1 \mathrm{H}, \mathrm{d}, J=8.1, \text { arom. }) ; 8.19(1 \mathrm{H}, \mathrm{t}, J=7.7, \\
\text { arom. }) ; 8.31(1 \mathrm{H}, \mathrm{d}, J=10,5,=\mathrm{CH}) ; 8.43(1 \mathrm{H}, \mathrm{s}, \mathrm{NH}) ; 11.92(1 \mathrm{H}, \mathrm{d}, J=10,5,=\mathrm{CH})\end{array}$ \\
\hline
\end{tabular}


Nowadays a lot of attention is devoted to the study of antiradical activity of various Schiff bases, e.g., thymol and carvacrol based imines [8] and 4-aminoantipyrine derivatives [9]. We were interested in finding out the possible antiradical activity of imines containing quinozolinone moiety. Antiradical activity for the synthesized compounds was detected with 2,2-diphenyl-1-picrylhydrazyl (DPPH) method. Unfortunately, most of the synthesized compounds $\mathbf{4}$ and $\mathbf{5}$ did not demonstrate any antiradical activity against DPPH. Only compounds $4 \mathbf{e}$ and 4f exhibited a weak scavenging activity against DPPH; unfortunately, the antiradical activity of imine containing substituents exact as for protocatechuic aldehyde $\mathbf{4 f}$ was surprisingly low in comparison with other compounds containing moiety of 3,4-dihydroxybenzaldehyde, e.g., caffeic acid esters or their hydrogenated derivatives are well-known antioxidants [10], [11]. Also compounds 5c and 5d demonstrated a slight antiradical activity; their antiradical activity is nearly $50 \%$ of the antiradical activity demonstrated by widely used antioxidant butylated hydroxytoluene (nearly $38 \%$ [12]). It seems that Schiff bases $\mathbf{4}$ and $\mathbf{5}$ containing quinolinone moiety are not promising antiradical agents.

TABLE 3

DPPH SCAVENGING ACTIVITY FOR THE QUINAZOLINONES 4 AND 5 AGAINST FREE RADICAL DPPH ${ }^{a}$

\begin{tabular}{|c|c|c|}
\hline Compound & $\begin{array}{c}\text { Inhibition of DPPH, } \\
\%\end{array}$ & Standard deviation \\
\hline $\mathbf{4 a}$ & -0.8 & 0.3 \\
\hline $\mathbf{4 b}$ & 0.2 & 0.2 \\
\hline $\mathbf{4 c}$ & -0.9 & 0.2 \\
\hline $\mathbf{4 d}$ & 0.1 & 0.2 \\
\hline $\mathbf{4 e}$ & 9.5 & 1.1 \\
\hline $\mathbf{4 f}$ & 8.0 & 0.5 \\
\hline $\mathbf{4 g}$ & 0.7 & 0.1 \\
\hline $\mathbf{4 h}$ & -0.5 & 0.3 \\
\hline $\mathbf{4 i}$ & -0.4 & 0.4 \\
\hline $\mathbf{4 j}$ & 0.0 & 0.1 \\
\hline $\mathbf{4 k}$ & 2.9 & 0.4 \\
\hline $\mathbf{4 l}$ & -0.5 & 0.0 \\
\hline $\mathbf{5 a}$ & 4.1 & 0.4 \\
\hline $\mathbf{5 b}$ & 4.9 & 0.1 \\
\hline $\mathbf{5 c}$ & 19.5 & 1.4 \\
\hline $\mathbf{5 d}$ & 14.9 & 0.4 \\
\hline
\end{tabular}

${ }^{a}$ The molar ratio of DPPH and quinazoline derivative $\mathbf{4}$ or 5 was 1:1.

\section{EXPERIMENTAL SECTION}

${ }^{1} \mathrm{H}$ NMR spectra were obtained on Bruker 300 spectrometer in DMSO- $\mathrm{d}_{6}$. The progress of the chemical reactions and the purity of products were monitored by TLC on silica gel plates (Merck $60 \mathrm{~F}_{254}$ ), using $\mathrm{CHCl}_{3}-\mathrm{CH}_{3} \mathrm{OH}-\mathrm{CH}_{3} \mathrm{COOH}$ (9:1:1) as eluent. The characteristics and ${ }^{1} \mathrm{H}$ NMR spectra of the compounds 4a-g and 5a-d are cited in Table 1 and Table 2. The absorption of the solutions (for DPPH test) was measured with Camspec M501 Single Beam Scanning UV/Visible spectrophotometer. The DPPH test was carried out as described previously [12]. The results are presented as the mean of two independent measurements \pm standard deviation. Each experiment was repeated three times for various concentrations of the solutions of Schiff base $\mathbf{4}$ or $\mathbf{5}$.

2,2-Dimethyl-5-[(2-(4-oxoquinazolin-3(4H)-ylamino)methylene]-1,3-dioxane-4,6-dione (5a), diethyl-2-(4oxoquinazolin-3(4H)-ylamino)methylene malonate (5b), ethyl2-cyanocarbonyl-(4-oxoquinazolin-3(4H)-ylamino) acrylate (5c), 3-[(4-oxoquinazolin-3(4H)-ylamino) methylene]pentane-2,4dione $(\mathbf{5 d})$.

3-Aminoquinazolin-4(3H)-one (1) (0.001 mol) and ethoxymethylene derivative 3a-d $(0.001 \mathrm{~mol})$ were stirred in ethanol $(5 \mathrm{ml})$ at room temperature for $3 \mathrm{~h}$ (for compound 3a) or refluxed in toluene $(5 \mathrm{ml}$ ) for $15 \mathrm{~h}$ (for compounds $\mathbf{3 b}$-d). Compound 5a was filtered off and recrystallized. The reaction mixtures containing compounds $\mathbf{5 b - d}$ were cooled, the precipitate filtered and recrystallized.

(Z)-3-(Arylideneamino)quinazolin-4(3H)-one (4a-f), (Z)-3(pyridin-3-ylmethyleneamino)quinazolin-4(3H)-one $(\mathbf{4 g})$.

A mixture of 3-aminoquinazolin-4(3H)-one (1) $(0.001 \mathrm{~mol})$ and aldehyde $2 \mathbf{a}-\mathbf{g}(0.001 \mathrm{~mol})$ was heated to reflux in ethanol $(5 \mathrm{ml}$ ) for $3 \mathrm{~h}$ (for compounds $\mathbf{2 a}, \mathbf{b}, \mathbf{d}, \mathbf{e})$ or $6 \mathrm{~h}$ (for compound 2c) or in toluene (5 $\mathrm{ml}$ ) for $3 \mathrm{~h}$ (for compound $\mathbf{2 f}$ ) or $8 \mathrm{~h}$ (for compound $\mathbf{2 g}$ ), then cooled, kept for $16 \mathrm{~h}$ in a refrigerator and filtered.

\section{REFERENCES}

[1] Rakhi, R., Abhinav, P. M. A Review on Biological Activity of Quinazolinones. International Journal of Pharmacy and Pharmaceutical Sciences, 2012, vol. 4, N 2, pp. 66-70.

[2] Panneer Selvam, T., Nijayaraj Kumar, P., Siva Kumar, A. Synthesis and Anti-oxidant Activity of Novel 6,7,8,9-tetrahydro-5H-5-(2'-hydroxy phenyl)-2-(4'-substituted benzylidene)-3-(4-nitrophenylamino)triazolo quinazoline Derivatives. Research in Biotechnology, 2010, N 1, pp. 38-48.

[3] Mohana Roopan, S., Nawaz Khan, F. Free Radical Scavenging Activity of Nitrogen Heterocyclics-quinazolinones and Tetrahydrocarbazolones. Indian Journal of Heterocyclic Chemistry, 2008, vol. 18, N 2, pp. 183-184.

[4] Kovalenko, S., Belenichev, I., Galitsa, V., Krivoshey, O. Synthesis of 2-(3,4-dihydro-3-oxo-2H-[1,2,4]triazino[4,3-c]quinazolin-4-yl)acetic Acid Derivatives as Potential Antioxidants in Nitrosative Stress Model. Farmacia, 2010, vol. 58, N 2, pp. 145-157

[5] Zhang, Y., Fang, Y., Liang, H., et. al. Synthesis and Antioxidant Activities of 2-oxo-quinoline-3-carbaldehyde Schiff-base Derivatives. Bioorganic and Medicinal Chemistry Letters, 2013, vol. 23, N 1, pp. 107-111. http://dx.doi.org/10.1016/j.bmcl.2012.11.006

[6] Shemchuk, L. A., Chernykh, V. P., Arzumanov, P. S. et al. Synthesis of $\quad \mathrm{N}$-(4-oxo-3,4-dihydroquinazolin-3-yl)succinimide and $\mathrm{N}$-(4oxoquinazolin-3-yl)succinamic Acid Derivatives Based Thereon. Russian Journal of Organic Chemistry, 2007, vol. 43, pp. 615-618. http://dx.doi.org/10.1134/S1070428007040215

[7] Rāviņa, I., Tetere, Z., Zicāne, D. Metoksibenzolidēnamino-3, 4-dihidrohinazolinonu sintēze. Latvijas Kïmijas žurnāls, 2009, Nr. 3, 231.-235.lpp.

[8] Deepak Kumar, B., Rawat, D. S. Synthesis and Antioxidant Activity of Thymol and Carvacrol Based Schiff Bases. Bioorganic and Medicinal Chemistry Letters, 2013, vol. 23, N 3, pp. 641-645. http://dx.doi.org/10.1016/i.bmcl.2012.12.001

[9] Sayed Alam, M., Choi, J.-H., Lee, D.-U. Synthesis of Novel Schiff Base Analogues of 4-Amino-1,5-dimethyl-2-phenylpyrazol-3-one and Their Evaluation for Antioxidant and Anti-inflammatory Activity. Bioorganic and Medicinal Chemistry, 2012, vol. 20, N 13, pp. 4103-4108. http://dx.doi.org/10.1016/j.bmc.2012.04.058

[10] LeBlanc, L. M., Paré, A. F., Jean-François, J., et al. Synthesis and Antiradical/Antioxidant Activities of Caffeic Acid Phenethyl Ester and Its Related Propionic, Acetic, and Benzoic Acid Analogues. Molecules, 2012, vol. 17, pp. 14637-14650. 
http://dx.doi.org/10.3390/molecules 171214637

[11] Gülçin, I. Antioxidant Activity of Caffeic acid (3,4-dihydroxycinnamic acid). Toxicology, 2006, vol. 217, N 2-3, pp. 213-220. http://dx.doi.org/10.1016/j.tox.2005.09.011

[12] Mierina, I., Tetere, Z., Zicāne, D. et al. Synthesis and Antioxidant Activity of New Analogs of Quin-C1. Chemistry of Heterocyclic Compounds, 2013, vol. 48, pp. 1824-1831. http://dx.doi.org/10.1007/s10593-013-1215-3

Zenta Tetere received her Dr. chem. degree in 1975. She has been working at the Faculty of Material Science and Applied Chemistry, Riga Technical University, where currently she is a Leading Researcher. Her current research interests include chemistry of heterocycles.

E-mail: zenta@ktf.rtu.lv

Daina Zicāne received her Dr. chem. degree in 1974. She has been working at the Faculty of Material Science and Applied Chemistry, Riga Technical University, where currently she is a Leading Researcher. Her current research interests include chemistry of heterocycles.

Address: Paula Valdena Str. 3, Riga, LV-1007, Latvia

E-mail: daina_zi@ktf.rtu.lv
Irisa Rāviņa obtained her Dipl. ing. degree in 1975. Further she has worked as an Engineer at the USSR Institute of Applied Biochemistry Olaine. Since 1981, Irisa Rāviņa has been working at the Faculty of Material Science and Applied Chemistry, Riga Technical University, where currently she is a Researcher. E-mail: irisara@inbox.lv

Inese Mierina received her Bc. and Mg. sc. ing. in Chemistry and Chemical Engineering from Riga Technical University (Faculty of Material Science and Applied Chemistry) in 2007 and 2009. Since 2009, she has been a doctoral student. She has been a Researcher (since 2009) at the Institute of Technology of Organic Chemistry (Riga Technical University, Faculty of Material Science and Applied Chemistry) and she has been a Lecturer (since 2011) at the Department of Biologically Active Compounds. She was a Research Assistant from 2005 till 2009 at the same department. Her research is focused on the synthesis of natural antioxidant analogues and plant material extracts as potential antioxidants for vegetable oils.

E-mail: inesem@ktf.rtu.ly

Inese Rijkure obtained her Dipl. ing. degree in 1969. Further she has worked as an Engineer at Riga Polytechnical Institute. I. Rijkure worked as a Laboratory Manager at the factory "Komutators" in 1978 - 1979. Since 1979, Inese Rijkure has been working at the Faculty of Material Science and Applied Chemistry, Riga Technical University, where currently she is a Researcher. E-mail: daina_zi@ktf.rtu.lv

Zenta Tetere, Daina Zicāne, Irisa Rāviṇa, Inese Mieriṇa, Inese Rijkure. Jaunu hinazolinonu atvasinājumu sintēze un to antioksidatīvās ipašĭbas

Hinazolīna ciklu saturošie savienojumi literatūrā ir ḷoti plaši pētīiti, jo tiem piemīt visdažādākā bioloǵiskā aktivitāte, taču ziṇu par hinazolīna atvasinājumu antioksidatīvām īpašībām literatūrā nav daudz. Publicēti atsevišḳi ziņojumi par hidroksilgrupas un sēra atoma klātbūtnes veicinošu ietekmi uz hinazolīna antioksidatīvo īpašību paaugstināšanu, tomēr publikācijā nav aprakstīti sistemātiskie pētījumi par hinazolīna cikla aizvietotāju un antioksidatīvās aktivitātes rādītāju savstarpējām likumsakarībām. Literatūrā publicēti dati par hinolīna rindas Šiffa bāzu augstiem antioksidatīvās aktivitātes rādītājiem. Hinolīna un hinazolīna ciklu strukturālā līdzība ḷauj uzskatīt arī hinazolīna rindas Šiffa bāzes par potenciāliem antioksidantiem. Minētie fakti, kā arī antioksidantu nozīmes pieaugums brīvo radikāḷu izraisīto procesu stabilizācijā rosināja darba autorus veikt dažu hinazolīn-4(3H)-onu atvasinājumu sintēzi un antioksidatīvās aktivitātes pētījumus. Par izejvielu eksperimentos izmantots 3-aminohinazolīn-4(3H)-ons, kas iegūts no antranilskābes hidrazīda un ortoskudrskābes trietilestera. 3-Aminohinazolīn-4(3H)-ons modificēts pie $\mathrm{N}(3)$ atoma ar aldehīdiem un etoksimetilēnatvasinājumiem, iegūstot $\mathrm{C}=\mathrm{C}$ un $\mathrm{C}=\mathrm{N}$ saiti saturošus savienojumus. Sintezēto savienojumu struktūras noteiktas ar ${ }^{1} \mathrm{H}$ KMR spektru palīdzību un pamatotas ar elementanalīzes datiem. Pārbaudīta publikācijā aprakstīto savienojumu, kā arī piecu agrāk sintezēto hinazolīna Šiffa bāzu antioksidatīvā aktivitāte.

Зента Тетере, Дайна Зицане, Ириса Равиня, Инесе Миериня, Инесе Рийкуре. Синтез новых производных хиназолинона и их антиоксидантные свойства

Соединения с хиназолиновым циклом очень широко исследованы, поскольку они обладают самой различной биологической активностью, в то же время сведений об их антиоксидантной активности в литературе крайне мало. Имеются сообщения авторов некоторых статьей о заместителях хиназолинового цикла, которые, по их мнению, придают молекуле антиоксидантную активность, например, гидроксильные группы и присутствие атома серы, но систематический анализ возможной закономерности структураактивность практически отсутствует. В литературе имеются данные о высокой антиоксидантной активности оснований Шиффа в ряду 2-оксохинолина. Структурное сходство циклов хинолина и хиназолина позволяет основания Шиффа 4-оксохиназолина также представлять потенциальными антиоксидантами. Всё вышеизложенное, а также рост значения и необходимость применять к антиоксидантам, стабилизирующие процессы, вызванные свободными радикалами, было стимулом для синтеза и изучения нами антиоксидантной активности некоторых производных хиназолин-4(3H)-онов. В качестве исходного вещества выбрали 3 -аминохиназолин-4(3H)-он, синтезированный из гидразида антраниловой кислоты и триэтилового эфира ортомуравьиной кислоты по литературной методике. Исходный аминохиназолон модифицировали при $\mathrm{N}(3)$ с образованием соединений с $\mathrm{C}=\mathrm{C}$ и $\mathrm{C}=\mathrm{N}$ связями в его реакциях с альдегидами и этоксиметиленсоединениями. Структуры синтезированных соединений определены с помощью спектров ЯМР ${ }^{1} \mathrm{H}$ и подтверждены с данными элементного анализа. Проверена антиоксидативная активность в публикации описанных соединений и пять ранее синтезированных Шиффовых оснований хиназолина. 